\title{
Kernos
}

Revue internationale et pluridisciplinaire de religion grecque antique

15 | 2002

Varia

\section{L'usage du mythe chez Aristote}

\section{Toula Vassilacou-Fassea}

Édition électronique
URL : http://journals.openedition.org/kernos/1366

DOI : 10.4000/kernos. 1366

ISSN : 2034-7871

\section{Éditeur}

Centre international d'étude de la religion grecque antique

Édition imprimée

Date de publication : 1 janvier 2002

ISSN : 0776-3824

\section{Référence électronique}

Toula Vassilacou-Fassea, «L'usage du mythe chez Aristote », Kernos [En ligne], 15 | 2002, mis en ligne le 21 avril 2011, consulté le 10 décembre 2020. URL : http://journals.openedition.org/kernos/1366 ; DOI : https://doi.org/10.4000/kernos.1366 


\section{L'usage du mythe chez Aristote}

Depuis Homère jusqu'à l'époque d'Aristote, le sens du mot $\mu \hat{v} \theta$ os a $e^{e} v o l u e^{-1}$. Dans le langage homérique, le terme pouvait désigner simplement la parole $^{2}$. Chez Aristote, le mot est chargé de nuances diverses sans pour autant perdre sa signification initiale.

Le Corpus d'Aristote comprend 71 occurrences du mot mûtbos ${ }^{3}$; les sens que nous avons rencontrés sont les suivants :

1) Récit sacré traditionnel invérifiable ou faux ${ }^{4}$,

2) Récit inventé non traditionnel $l^{5}$,

3) Fable ésopique ${ }^{6}$,

4) Récit mensonger des magiciens ou des femmes ${ }^{7}$,

5) Assertion fausse ${ }^{8}$,

1 Sur l'évolution du sens du mot mâthos, $c f$. J. Desautels, "Mythe et politique: un voyage au royaume de l'ambigu", in Fr. Jounn, A. Motтe (éds), Mythe et Politique. Actes du Colloque de Liège 14-16 septembre 1989, Paris, 1990, p. 125-137.

2 M. Detienne, L'invention de la mythologie, Paris, 1981, p. 93. Cf. aussi L. CoulouBARITSIs, "La présence du mythe dans la pensée grecque des origines à Platon", Ludus Magistralis 61 (1985-1986), p. 13-32.

3 Dont cinquante sont dans la Poétique, une dans le De Anima, une dans le De Coelo, six dans l'Historia Animalium, une dans le De Motu Animalium, deux dans la Métaphysique, deux dans les Météorologiques, une dans le De Mundo, une dans la Politique, une dans les Problèmes et cinq dans les fragments.

4 Le mythe, pris dans le sens du récit sacré, c'est-à-dire, qui met en scène des dieux, des héros, des hommes héroïsés du passé, des personnages légendaires, apparaît au moins dans les passages suivants: Métaph., A, 2, 982b; 19 et L, 9, 1074b 1-3; Pol. VII, 17, 1336a 28-32; Poétique, 9, 1452a 1-11; Hist. anim. VI, 35, 580a 17 et sq; et VIII, 32, 619a 18-20; Mouvements anim. III, 699a 28-31; De Coelo II, 1, 284a 18-23; dans l'œuvre pseudoaristotélicienne De Mundo, 401b 22 et aussi dans quatre des cinq occurrences qui se rencontrent dans les fragments : fr. 142, 1.6 (p. 120); fr. 163, 1.9 (p. 130);fr. 172, 1.4 (p. 134); et fr. $573,1.6$ (p. 355) Rose.

5 Fr. 573 (p. 355) Rose.

6 Météor. II, 3, 356b 9-18. De même, le mot mytharia, désignant les fables ésopiques, apparaît dans le fr. n 1.5 sq., TLG, Fragmenta varia, Category 1, treatise title 4.

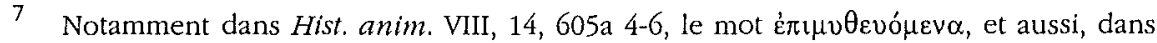
la même œuvre, VI, 578b 23-26, le mot $\mu v \theta 0 \lambda \circ \gamma o v \mu \varepsilon ́ v \omega v$ est chargé du même sens.

8 Hist, anim. VI, 31, 579b 2-4; et VI, 35, 580a 14-16. 
6) Mensonge? , et

7) Comme partie de la tragédie, il a le sens de l'imitation de l'action ${ }^{10}$.

Le mythe pour Aristote relève d'un discours qui n'est pas nécessairement un récit. De ce point de vue, le mûthos est une espèce de logos, mais un logos invérifiable. D'ailleurs, les deux vocables se présentent comme des synonymes dans quelques $\operatorname{cas}^{11}$.

La notion de mythe est ambiguë; le mot mythe suffit à disqualifier une parole et à la classer dans la catégorie des paroles inventées, fausses voire mensongères. D'autre part, le mythe, en tant que récit sacré, est considéré par Aristote comme digne d'attirer son attention; aussi l'interprète-t-il pour dégager les vérités, ou les messages qu'il contient. Pour cette raison, le récit sacré fera l'objet de notre petit exposé.

Même si Aristote s'est abstenu de créer des mythes, il n'a pas moins fait, lui aussi, un usage assez abondant des récits traditionnels. Nous pouvons distinguer deux types d'usage : celui qui prend en considération le contenu et celui qui se rapporte à un élément purement formel.

Dans la première catégorie, celle où le contenu des mythes est concerné, nous pouvons intégrer les mythes qui sont utilisés à titre d'exemple ${ }^{12}$ - ces cas sont les plus nombreux - et les mythes qui sont directement traités. Ces derniers sont les plus intéressants.

Les mythes qui sont utilisés pour des raisons purement formelles le sont essentiellement à titre d'exemple. Ces emplois se trouvent surtout dans la Poétique et la Rbétorique. Ici, le contenu n'intéresse pas directement le philosophe; à travers des exemples, tirés de la poésie ou de l'épopée homérique $^{13}$, il articule des règles qui ont comme but soit la création de la bonne tragédie, soit la formation d'un discours rhétorique efficace.

9 Histoire des animaux VII, 11, 597a 7-9.

10 Sur le sens du mot mâtbos dans la Poétique, cf., entre autres, E. Downing, "Ofov

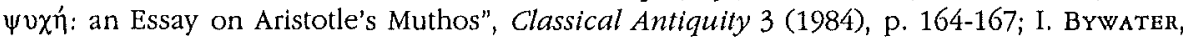
Aristotle on the Art of Poetry, Oxford, 1909; S.H. BuTCHer, Aristotle on Poetry and Music, Londres, 1911; G.F. Else, Aristotle's Poetics, Harvard, 1957; D.W. Lucas, Aristotle: The Poetics, Oxford, 1968; I. During, Aristoteles, Darstellung und Interpretation seines Denkens (traduit en grec par P. Kotziapanteli), Athènes, 1994, p. 273.

11 Le mot logos est associé ou même équivalent au mot mûthos au moins quinze fois dans le corpus d'Aristote; notamment dans les ceuvres suivantes: Rbét., 1393a 32; $1393 \mathrm{~b} 8$ et 12; 1394a 2, 3 et 5; Problèmes, 916b 26-35 (trois occurrences); De Coelo II, 1, $284 \mathrm{a} 21$. Politique VII, 17, 1336a 30; Poétique, 1449b 8; 1455a 35; 1455b 17; 1460a 26.

12 La fréquence la plus grande d'exemples tirés de la mythologie se rencontre dans les traités de philosophie morale, c'est-à-dire, dans l'Éthique à Eudème et l'Éthique à Nicomaque.

13 J. LABARBe, "Deux citations homériques d'Aristote", in Aristotelica. Mélanges offerts à Marcel De Corte, Liège, 1985 (Cabiers de Pbilosophie ancienne, 3), p. 226, a relevé à peu près 150 citations d'Homère dans le Corpus aristotélicien. 
Mais nous allons nous borner ici aux mythes qui sont directement traités, et qui peuvent mieux éclairer la conception du philosophe sur la valeur de la tradition mythique.

Selon la conception qui s'exprime dans la Métapbysique ${ }^{14}$, le mythe s'apparente à la philosophie par différents traits. En premier lieu, parce qu'il est constitué de choses merveilleuses, il provoque l'étonnement ( $\delta$ ì̀ tò $\theta \alpha v \mu \alpha ́ \zeta \varepsilon ı$ ) et pousse les penseurs à acquérir la sagesse (sopbia), en sorte que, dit le Stagirite, «l'amateur des mythes est en quelque manière amateur

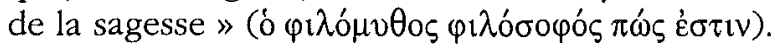

L'étonnement $^{15}$ est donc considéré par Aristote ${ }^{16}$ comme la motivation nécessaire qui incite les penseurs à la recherche de la sagesse ${ }^{17}$. Le rapport du mythe avec l'étonnement consiste dans le fait qu'il est constitué de choses merveilleuses, c'est-à-dire, des choses qui, grâce à des attributs exceptionnels, dépassent la mesure humaine. Le mythe suscite l'étonnement en mettant en scène des êtres surnaturels, en présentant un au-delà auquel est étrangère l'expérience immédiate de l'homme, en intégrant souvent l'intelligible dans le monde sensible; c'est cela que font les mythes sur les dieux, les héros et les défunts. Mais ce n'est pas tout: le second trait, par lequel le mythe s'apparente à la philosophie, concerne le contenu même du mythe, lequel peut avoir un noyau vrai, un fondement inital, porteur de vérité. Donnons la parole au philosophe:

Une tradition transmise de l'antiquité la plus reculée ( $\pi \alpha \rho \dot{\alpha} \tau \hat{\omega} v$ d $\rho \chi \alpha \alpha i \omega v$

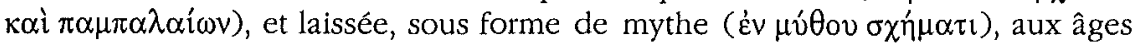
suivants, nous apprend que les premières substances sont des dieux, et que le

14 Métaph. A, II, 982b 11-19, trad. J. Tricot, Paris, Vrin, 1986, où le sujet traité est la définition de la sagesse. Le passage mérite d'être cité : "Qu'elle ne soit pas, d'autre part, une science poétique, c'est ce que montre aussi l'histoire des plus anciens philosophes.

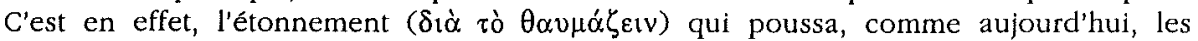
premiers penseurs aux spéculations philosophiques. Au début, leur étonnement porta sur les difficultés qui se présentaient les premières à l'esprit; puis, s'avançant ainsi peu à peu, ils étendirent leur exploration à des problèmes plus importants, tels que les phénomènes de la Lune, ceux du Soleil et des Étoiles, enfin la genèse de l'Univers. Or, apercevoir une difficulté et s'étonner, c'est reconnaître sa propre ignorance - c'est pourquoi même l'amateur des mythes est, en quelque manière, amateur de la Sagesse (ó $\varphi \imath \lambda o ́ \mu v \theta o \zeta$

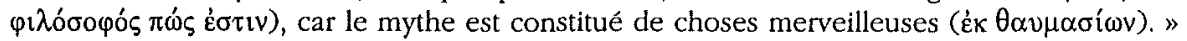

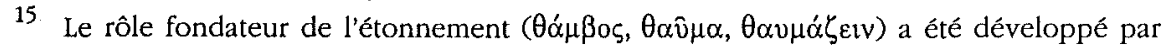
A. Motre, "L'expression du sacré dans la religion grecque", L'expression du sacré dans les grandes religions, Louvain-La-Neuve, 1986 (Homo religiosus, 3), p. 170-172.

16 Platon aussi considère le $\theta \alpha v \mu \alpha \zeta \xi \mathfrak{~} \mathbf{v}$ comme l'acte originel de la philosophie; dans

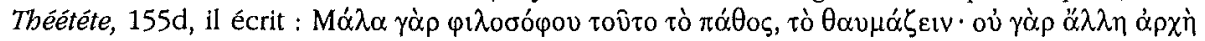

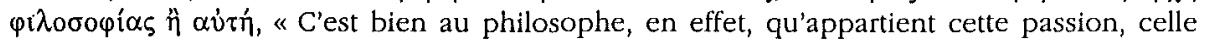
de s'étonner, car il n'y a pas d'autre acte originel de la philosophie que celui-là ».

17 M.D. YAFFE, "Myth and 'Science' in Aristotle's 'Theology", Man and World 12 (1979), p. 70-88, croit que dans le passage précédent de la Métaphysique, Aristote parle indirectement de la relation entre la science et le mythe. 
divin embrasse la nature entière. Tout le reste de cette tradition a été ajouté plus tard, sous une forme mythique $(\mu v \theta 1 \kappa \hat{\omega} \varsigma)$, en vue de persuader les

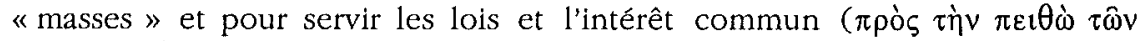

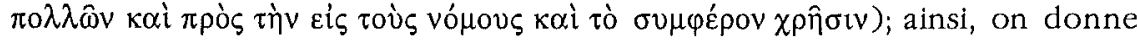
aux dieux la forme humaine, ou on les représente semblables à certains animaux, et on adjoint toutes sortes de précision de ce type. Si l'on sépare du récit son fondement initial ( croyance que toutes les substances premières sont des dieux, alors on pensera que c'est là une assertion vraiment divine. Alors que, selon toute vraisemblance, les divers arts et la philosophie ont été, à plusieurs reprises, développés aussi loin que possible et chaque fois perdus, ces opinions sont, pour ainsi dire, des reliques de la sagesse antique (ốov $\lambda$ eí $\psi \alpha \nu \tau \alpha$ ) conservées jusqu'à notre temps. Telles sont donc les réserves sous lesquelles nous acceptions la tradition de nos pères et de nos plus anciens devanciers ( $\dot{\eta} \mu \dot{\varepsilon} v$

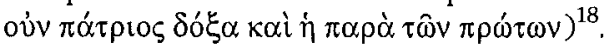

Les conclusions que nous pouvons tirer des deux passages précédents sont essentielles pour révéler l'attitude d'Aristote envers la mythologie : 1) Il présente un attachement sincère à la tradition; son attitude manifeste une réelle continuité entre les traditions mythiques et la philosophie ${ }^{19}$; 2) Il y a une différence entre la forme de mythe $(\sigma \chi \hat{\eta} \mu \alpha \mu v \dot{\theta}$ ov) et l'intuition mentale essentielle comprise dans le récit mythique ( $\tau$ ò $\pi \rho \omega \hat{\tau} \tau$ ) ), qu'on doit prendre en considération.

Aristote, à la différence de Platon, n'exploite pas les mythes pour susciter le sentiment du sacré. Mais le mythe comme récit sacré est en rapport direct avec la religion ${ }^{20}$, puisqu'il se réfère au monde du sacré. Quel est toutefois le noyau vrai compris dans les mythes qui mettent en scène les dieux, et quels sont les éléments qui constituent l'ajout mythique? En ce qui concerne les dieux, le noyau vrai consiste, comme nous l'avons vu auparavant, en ce que «les premières substances sont des dieux et que le divin embrasse la nature entière ». Le reste est l'ajout mythique, véhiculé par les théologiens. Par

18 Métaph. $\Lambda, 9,1074 \mathrm{~b}$ 1-14, trad. J. Tricot, légèrement modifiée.

19 Comme le souligne justement L. Brisson, Sauver les mytbes, Paris, 1996, p. 38.

20 Sur le caractère religieux du mythe, $c f$. J. RudHARDT, Du mytbe, de la religion grecque et de la comprébension d'autrui, Genève, 1981 (Cabier Vilfredo Pareto). Les réserves qui ont été exprimées sont en rapport avec le degré de sacralisation des mythes grecs; selon R. Bodéüs, Aristote et la théologie des vivants immortels, Québec/Paris, 1992, p. 127128 , « les mythes grecs sont peut-être moins sacrés que ceux des autres civilisations, plus proches du conte populaire. Mais ils paraissent, comme eux, avoir une signification que la raison est appelée à déceler sous la fantaisie. Et on a cru voir, chez Aristote lui-même, une attitude qui témoigne d'une conviction rationaliste semblable ». Selon P. WATHELET, "Homère du mythe à la mythologie", in Le mythe, son langage, son message, Louvain-laNeuve, 1983, p. 209-223, dès Homère, les récits mythiques ne sont plus d'essence religieuse et sont traités comme de l'histoire. Selon H. Doerrie, "Der Mythos im Verständnis de Antike, II: Von Euripides bis Seneca", Gymnasium 73 (1966), p. 44-62, le mythe n'est pas seulement religieux; c'est un miroir de l'Antiquité en général et de l'homme antique époque par époque. 
conséquent, du strict point de vue de la vérité, l'anthropomorphisme et le zoomorphisme des dieux sont à rejeter aussi bien que les récits qui veulent que les dieux consomment du nectar et de l'ambroisie ${ }^{21}$. C'est la même attitude qu'on doit adopter envers les récits qui représentent Atlante soutenant le Monde ${ }^{22}$ ou la jalousie des dieux envers les mortels qui excellent en sagesse $^{23}$. De même sont à rejeter les conceptions des théologiens selon lesquelles toutes choses sont nées de la $\mathrm{Nuit}^{24}$, ou celles qui donnent la royauté du Monde à Zeus et non à la Nuit, au Ciel, au Chaos ou à l'Océan, qui sont apparus les premiers. Cette opinion traduit l'idée inacceptable que les divinités qui gouvernent le Monde ne sont pas toujours les mêmes ${ }^{25}$.

21 Métaph. Ir, 4, 1000a 8-18, trad. J. Tricot : « Les contemporains d'Hésiode et tous les théologiens se sont, en vérité, souciés uniquement de ce qui pouvait entraîner leur propre conviction, mais ils ont négligé de penser à nous. Considérant, en effet, les principes comme des dieux et comme d'origine divine, ils disent que les êtres qui n'ont pas goûté le nectar et l'ambroisie sont mortels: c'est là évidemment employer des mots dont le sens ne peut que leur être familier; pourtant ce qu'ils ont dit de l'application même de ces causes nous dépasse. Si c'est en vue de plaisir, en effet, que les immortels touchent à ces breuvages, le nectar et l'ambroisie ne sont en rien causes de leur existence; et si c'est en vue de maintenir leur être, comment seraient-ils éternels, tout en ayant besoin de nourriture? "

22 De Coelo II, I, 284a 18-23, trad. J. Tricot : " C'est pourquoi il ne faut pas non plus ajouter foi au mythe ( $\tau$ òv $\tau \hat{\omega} v \pi \alpha \lambda \alpha t \hat{\omega} v \mu \hat{v} \theta 0 v$ ) antique qui assure que le Monde a besoin de quelque Atlante pour se soutenir. Ceux qui ont composé cette fable ( $\lambda$ ó $o v$ ) semblent bien adopter le même raisonnement que les penseurs plus récents : concevant tous les corps de la région supérieure comme ayant un poids et étant de nature terreuse, ils font reposer le Monde, d'une manière fausse $(\mu v \theta i \kappa \hat{\omega} \varsigma)$, sur une nécessité résidant dans une âme. "

23 Métaph. A, 2, 982b $32-983 a$ 5, trad. J. Tricot, où le sujet traité est la sagesse : « Si donc il y a quelque vérité dans ce que racontent les poètes, et si la jalousie est naturelle à la divinité, c'est bien dans ce cas qu'elle devrait, semble-t-il, surtout s'exercer, et tous ceux qui excellent dans ce savoir auraient un sort misérable. Mais il n'est pas admissible que la divinité soit jalouse (disons même, avec le proverbe, que les poètes sont de grands menteurs), et on ne doit pas non plus penser qu'une autre science puisse surpasser cellelà en dignité. »

24 Métaph. $\Lambda, 6,1071 \mathrm{~b} 25-28$, trad. J. Tricot : «Cependant, soit qu'on suive l'opinion des théologiens, qui font naître toutes choses de la Nuit, soit qu'à l'exemple des physiciens, on dise que toutes choses étaient confondues, c'est bien la même impossibilité qu'on retrouve. " Aristote a ici en vue Orphée, fr. B12 Diels-Kranz (vol. I, p. 10); Hésiode, Travaux et Jours, 17, et Théogonie, 116 sq; ARISTophane, Ois., 693; Épiménide, fr. B5 DielsKranz (vol. I, p. 33); Musée, fr. B14 Diels-Kranz (vol. I, p. 25), Pour les physiciens, ANAXAgore, fr. B1 Diels-Kranz (vol, Il, p. 32).

25 Métaph. N, 4, 1091b 4-8, trad. J. Tricot : « Les anciens poètes sont du même sentiment, en ce qu'ils donnent la royauté et le gouvernement non pas aux êtres qui sont apparus les premiers dans le temps, comme la Nuit et le Ciel, ou le Chaos, ou l'Océan, mais à Zeus. Cependant si ces poètes en viennent à s'exprimer ainsi, c'est seulement parce que, d'après eux, les divinités qui gouvernent le Monde ne sont pas toujours les mêmes. » 
Tous ces récits sont un ajout propre à l'expression mythique de la pensée et qui se développent autour d'un noyau vrai. Les récits mythiques qui représentent Zeus, Athéna, Héra, Aphrodite, etc., sous une forme anthropomorphe de même que Létô sous la forme d'une louve ${ }^{26}$, ou Héraclès accomplissant autant d'exploits, sont donc considérés comme fictifs, ce qui ne veut pas dire que ces êtres n'existent en aucune façon aux yeux d'Arstote; au contraire, il considère que le divin embrasse la nature entière. Dans le $D e$ Coelo, il dit :

Tous les hommes, en effet, se font une notion des dieux, et tous, tant qu'ils sont, Grecs et Barbares, qui croient à l'existence des dieux, s'accordent à localiser la divinité dans la région la plus haute, rattachant évidemment ainsi à l'immortel ce qui est immortel, et regardant toute autre supposition comme

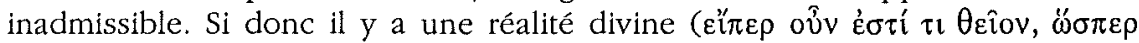
है $\sigma \tau \imath$ ), comme de fait il y en a certainement une, les indications que nous venons de donner sur la première substance corporelle étaient bien fondées ${ }^{27}$.

L'existence du divin n'est donc pas mise en cause ${ }^{28}$, même si les hommes représentent dans leurs mythes les dieux dotés d'une certaine forme ${ }^{29}$. La tâche du philosophe consiste en l'occurrence à interpréter les mythes pour élucider le mécanisme rationnel qui a conduit l'auteur à la création du mythe, et à puiser la vérité incluse dans le récit imagé. Telle est la méthode que suit Aristote dans son approche des mythes. Elle débouche sur un constat tantôt positif, tantôt négatif. Le message du mythe est considéré comme positif quand il satisfait aux exigences de la raison. Ainsi y a-t-il un noyau vrai dans le mythe qui représente Zeus capable de mouvoir l'univers, tout en étant luimême immobile et hors de l'univers. "Car on aboutirait à une absurdité si le principe du mouvement était interne. C'est pourquoi, si l'on adopte cette opinion, on doit penser qu'Homère a eu raison de dire : 'Vous n'amènerez

26 Hist. anim., 6, 35, 580a 17 sq.

27 De Coelo I, 270b 5-11, trad. J. Tricot.

28 Sur les principes qui régissent la pensée d'Aristote sur dieu et le divin, $c f$. C. Natali, Cosmo e divinità. La structura logica della teologia aristotelica, L'Aquila, 1974, (Méthodos-Collana di studi filosofici, 6). Sur la causalité de dieu, sa perfection divine, la connaissance qu'il a du monde, la bonté et l'amour de dieu pour les hommes et la Providence divine, $c f$. A. MANsion, "Le Dieu d'Aristote et le Dieu des chrétiens", in La philosopbie et ses problèmes. Mélanges R. Jolivet, Lyon, 1960, p. 21-44. Une analyse des "vues théologiques " d'Aristote est opérée par J. PÉPIN, Idées grecques sur l'bomme et sur Dieu, Paris, 1971, cf. p. 207-248, où il examine les textes : a) De Coelo I-II; b) Éth. Eudème VII-VIII; c) Éth. Nicomaque X, 7-9; d) Métaph., A, 7-9. Cf. aussi R Bodéüs, "En marge de la "théologie » aristotélicienne", $R P b L 73$ (1975), p. 5-33, où l'auteur propose une appréciation des pages précédentes de J. Pépin.

29 B. Dumoulin, Recherches sur le premier Aristote (Eudème, De la Pbilosopbie, Protreptique), Paris, 1981; Aristote est considéré comme passant d'une foi dans les vérités révélées par les traditions religieuses à l'adoption d'un rationalisme rigoureux. 
pas du ciel à la terre Zeus, qui est au-dessus de tous, quelque peine que vous preniez : accrochez-vous tous, dieux et déesses ${ }^{30}$. Car ce qui est absolument immobile ne peut être mû par rien $»^{31}$.

De même, le Stagirite essaie de séparer le récit mythique de son fondement initial, pour dégager une vérité sur la physique, dans le passage suivant des Météorologiques : «Quand, en effet, le Soleil s'approche, le courant de la vapeur monte; quand il s'éloigne, le courant de l'eau coule vers le bas. Et cela

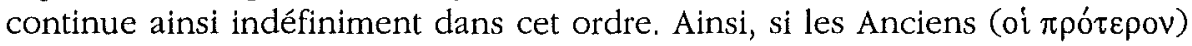
ont parlé à mots couverts de l'Océan, peut-être voulaient-ils désigner par là le fleuve qui coule circulairement autour de la Terre $»^{32}$.

À part la physique, le domaine de l'éducation est enrichi par les mythes. Dans la Politique, le philosophe, se référant à la fonction éducative de la flûte, accorde une pertinence au mythe qui dit qu'Athéna a abandonné la flûte après l'avoir inventée ${ }^{33}$. La cause est donnée à la suite du passage : «On n'a donc pas tort de dire que la déesse fit cela, parce qu'elle fut fâchée d'avoir son visage enlaidi. Néanmoins il est plus vraisemblable que c'est parce que l'étude de l'aulos n'enrichit en rien l'intelligence; or c'est à Athéna que nous attribuons la science et l'art $»^{34}$.

Donc, le refus de la flûte par la déesse pour des raisons esthétiques est bien possible. Athéna ne tolérait pas une déformation de son visage. Mais, la seconde raison est beaucoup plus vraisemblable : la flûte ne sert en rien au raisonnement. Dès lors, le mythe des Anciens, ayant de la pertinence et étant chargé d'un sens symbolique, se transforme, chez Aristote, en un récit rationnel.

En outre, le domaine de la morale est souvent concerné par les mythes. Il est probable qu'Aristote admettait l'existence d'un temps héroïque qui aurait

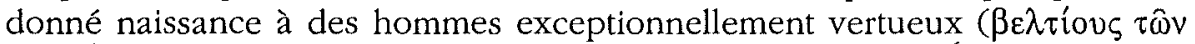
vôv $)^{35}$ et constituant des modèles pour les hommes actuels ${ }^{36}$. Cette conviction justifie l'usage du grand nombre de mythes qu'on trouve dans les traités de morale. D'ailleurs, quand le philosophe traite des notions morales, il ne vise pas à une approche spéculative du sujet; son but est le bien agir des citoyens.

Les mythes peuvent aussi être porteurs d'un message politique. Les institutions politiques sont décrites par les mythes où le roi prestigieux incarne en

\footnotetext{
30 Iliade VIII, 21-22 et 20, intervertis.

31 Mouv. anim. IV, 699b 34 - 700a 5, trad. P. Louis, Belles Lettres, 1973 (C.U.F.). Sur ce passage, $c f$. Brisson, o.c. (n. 19), p. 59 sq. et J. Pépin, Mythe et allégorie, Paris, 1976, p. 123

32 Météor. I, 9, 347a 4-8, trad. J. Tricot, Paris, Vrin, 1955, légèrement modifiée.

33 Pol. VIII, 1341b 2-4, trad. J. Aubonnet, Belles Lettres, 1989 (C.U.F.).

34 Ibid., 4-8.

35 Poétique II, 1448a 18

36 L'idée est developpée par BoDÉüs, o.c. (n. 20), p. 147-148.
} $s q$. 
lui-même la loi. Agamemnon en est le modèle. Mais tous les mythes qui dans le Corpus aristotélicien sont relatifs à l'éducation, à la moralité et à la politique ne visent qu'à la transmission d'une paideia commune à tous, destinée à cimenter leur communauté.

En ce qui concerne l'origine des mythes, le philosophe ne nous laisse nulle part supposer qu'ils véhiculent une certaine inspiration divine. Selon R. Bodéüs, les mythes relèvent pour Aristote de la pure fiction ${ }^{37}$. Le philosophe a expliqué la manière dont ces conceptions avaient été adoptées par les Anciens : les Arts et la Philosophie avaient été développés aussi loin que possible, mais à plusieurs reprises leur enseignement s'est perdu. Aussi les mythes conservent-ils les reliques de la sagesse des générations précédentes, à qui le philosophe attribue la caractérisation de "l'assertion divine ", analogue à la parole divine se servant d'une hyperbole. Telle est la tradition parvenue des Anciens et c'est cette raison qui la rend digne d'être respectée, selon Aristote ${ }^{38}$.

Or, Aristote ne fonde pas ses conclusions sur les mythes. Même si quelques-uns d'entre eux sont porteurs d'une certaine sagesse, ils appartiennent au langage invérifiable, et le philosophe exprime des réserves à leur égard comme en témoigne le vocabulaire qu'il utilise. D'ailleurs, la connaissance pour Aristote n'est que scientifique. Il ne prouve pas ses spéculations philosophiques par les mythes. Même s'il considère qu'il y a une continuité entre la tradition mythique et la philosophie, au moins en ce qui concerne les dieux, c'est-à-dire que la philosophie est, en quelque manière, le prolongement de la tradition mythique, il ne nous laisse pas supposer que le langage mytho-poétique est suffisant pour faire avancer la science. Il l'utilise pour illustrer sa pensée dans la majorité des cas.

Cela ne veut pas dire qu'il ne respecte pas la tradition, surtout celle qu'il attribue aux Anciens; au contraire, elle fait, dans quelques cas, l'objet de sa recherche, et il lui reconnaît une certaine pertinence. Aristote étant philo$m \hat{u} t h o s$, comme il l'avoue dans un fragment ${ }^{39}$, il combine l'amour pour les mythes avec la pensée rationnelle.

Toula VASSILACOU-FASSEA

37 Ibid., p. 134-149.

38 M. Mesuin, Pour une science des religions, Paris, 1973, p. 24, écrit : " C'est à mon sens, Aristote qui a le plus intelligemment expliqué la fonction étiologique des mythes: ces derniers n'indiquent pas les causes premières et logiques, les aitiai, mais seulement les causes dans la mesure où elles sont des archai, des commencements; non pas les causes immédiates mais les éléments primordiaux (Métaph. $\Delta, 1,1013 \mathrm{a}$ ). Ainsi l'effort pour remonter, par le truchement d'un récit mythique, hors du temps historique est d'abord un effort pour saisir l'essence même du divin. Dans tout langage mythique, l'ancienneté est synonyme d'essence, et les diverses généalogies, filiations, naissances, ne sont qu'un langage pour définir des relations causales entre les êtres et les choses, et pour préciser leurs rapports, soit dialectiques, soit de subordination."

39 Fr. 618, Aristotelis opera, éd. Academia Regia Borussica, Berolini, 1870 (vol. V

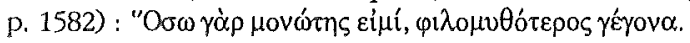

